\title{
Ultrasonography in Early Diagnosis and Follow up of Amniotic Fluid Abnormalities for Assessment of the Fetal Growth and Fetal Wellbeing
}

\author{
Ali Abdel Fattah Elsayed, ${ }^{1}$ MD, Salah Mohammed El Rays, ${ }^{1}$ MD.
}

* Corresponding Author:

Ali Abdel Fattah Elsayed

alyhelilamro@gmail.com

Received for publication January 20, 2021; Accepted February 16, 2021; Published online February 16, 2021.

Copyright 2021 The Authors published by Al-Azhar University, Faculty of Medicine, Cairo, Egypt. All rights reserved. This an open-access article distributed under the legal terms, where it is permissible to download and share the work provided it is properly cited. The work cannot be changed in any way or used commercially.

doi: 10.21608/aimj.2021.53808.1374.

${ }^{1}$ Radiology Department, Faculty of Medicine, Al-Azhar University.

\begin{abstract}
Background: The US (including Doppler \& 4D) is a simple method for early detection of amniotic fluid abnormalities to evaluate the fetal wellbeing and congenital lethal and non lethal anomalies.

Aim of the study: Study aims to the use of the ultrasonographic early diagnosis and follow up of amniotic fluid abnormalities for evaluation the fetal wellbeing, fetal growth and early warning signs of fetal distress to determine the suitable time for delivery.

Patients and Methods: This study was performed between November 2019 and July 2020 at Obied Hospital-KSA. The study was carried on 80 cases presented with amniotic fluid abnormalities.

Results: Out of 3220 pregnant females attending the outpatient clinic of the Gynecology and Obstetrics Department in ObH, 80 (2.48\%) females with amniotic fluid abnormalities were selected. The maternal age ranged from 19 to 40 years. The true gestational age ranged from 13 to 40 weeks. The results show that there were significant positive correlations between amniotic fluid abnormality and fetal congenital anomalies, fetal growth disturbance \& fetal wellbeing.I $n$ our study, the incidence of congenital anomalies was 44 cases out of 80 (55\%). Lethal anomalies was seen in 20 cases (45.45\% of congenital anomalies), non lethal in 24 cases $(54.54 \%)$. Our results demonstrate that there are different etiologies causing Amniotic fluid abnormalities.

Conclusion: Despite $45 \%$ of idiopathic etiology, we concluded that congenital anomalies should be carefully searched on whenever amniotic fluid abnormalities were detected. Full anomaly scan and close antenatal follow up are recommended.
\end{abstract}

Keywords: Ultrasonography; amniotic fluid; fetal wellbeing; fetal growth; congenital...

Disclosure: The authors have no financial interest to declare in relation to the content of this article. The Article Processing Charge was paid for by the authors.

Authorship: All authors have a substantial contribution to the article.

\section{INTRODUCTION}

Amniotic fluid measurement is mandatory as the amniotic fluid abnormalities occur in about 7 percent of pregnancies. ${ }^{1}$

Oligohydramnios is considered a feature of chronic hypoxemia.

Abnormalities associated with polyhydramnios involving the skeletal, gastrointestinal \& central nervous system also can occur. ${ }^{2}$

Fetal causes of oligohydramnios include fetal urinary tract anomalies, such as renal agenesis,

polycystic kidneys, or any urinary obstructive lesion (e.g., posterior urethral valves). Maternal causes can cause oligohydraminos. ${ }^{3}$
In polyhydramnios, the ultrasound is a simple tool to full anatomy scanning. ${ }^{4}$

In oligohydramnios, the ultrasound is also a simple tool to perform serial measurements during the pregnancy. ${ }^{5}$

The fetal growth abnormality is seen in cases of placental insufficiency, if rupture of membranes or urinary tract abnormalities are absent. ${ }^{4}$

\section{PATIENTS AND METHODS}

Type of the study: Prospective study (Observational prospective study) was conducted between November 2019 and July 2020. It was carried out at Obeid Hospital KSA on "3220" pregnant women who attended the outpatient clinic at $\mathrm{ObH}$, twodimensional ultrasound, $4 \mathrm{D}$ ultrasound were done to 
assess the fetal wellbeing, amniotic fluid index and TIFFA (targeted imaging for fetal anomalies).

Duration of follow up: during the 2nd and $3 \mathrm{rd}$ trimester.

Inclusion criteria: Patients aged between 19 and 40 years, viable pregnancies and gestational age from 16 weeks till the time of delivery with oligo or polyhydramnios.

Exclusion criteria: rupture of membranes (PROM); exclusion of postmaturity \& maternal causes (drug usage)

Collecting data: Out of 3220 Pregnant females coming for routine antenatal screening in the outpatient clinic of the Gynecology \& Obstetrics department in SGH or for confirmatory advice from other centers. 80 pregnancies with AF abnormalities were found. In the outpatient clinic all cases were evaluated by 2D US and those with suspected fetal abnormality, any previous fetal congenital anomaly or any strong positive family history were referred to the department of radiology. For dedicated cases, anomaly scan \& 4 D US were done.

Radiological studies: Every patient eligible for the study is submitted for a detailed ultrasound examination by two-dimensional ultrasound. Fetal gestational age was calculated according to date of LMP, then US at time of examination by measuring BPD, HC, FL and AC. Expected fetal weight (EFW) by Hadlock's formula (abdominal circumference, biparietal diameter, and femur length). AFI, targeted imaging for fetal anomalies and 4D.

The AFV were measured by three methods; the subjective method, largest pocket (LVP) and AFI

Outcome data: Primary outcome: cases with amniotic fluid abnormalities with or without anomalies.

Secondary outcome: the cases were divided into three groups.

Group A: oligohydramnios \& anhydramnios: diagnosed when the LVP is below 2 and the AFI is below 5 . They were 34 pregnancies.

Group B: less than average: diagnosed when the AFV below the lower normal by one parameter and normal by the other. they were 8 pregnancies.

Group C: polyhydramnios: the LVP is above 8 and the AFI is above or equal to $25 \mathrm{~cm}$. They were 38 pregnancies

History: Personal history included: Name, Age, address, telephone number\& mobile number, LMP, obstetric code, EDD, general disease \& medications, pregnancy associated diseases, any risk factor, previous baby with problem \& consanguinity.
Technique of examination \& study design: Ultrasound examination using US machine: Philips machine (for women health care) (Netherland)

In this a transadominal convex array volume transducer with frequency range $3.0-5.0 \mathrm{MHz}$ was employed for 2D US and another convex array volume transducer with frequency range 5.0-6.0 $\mathrm{MHz}$ to acquire the 3D\&4D volume data set. Full 2D (combined gray-scale and color Doppler studies) was done in all cases.

Amniotic fluid was measured using the subjective method, largest vertical pool (LVP) \& the four quadrants amniotic fluid index (AFI), all were used in order to avoid the intra- and inter observer variations.

Fetal full anomaly survey according the gestational age was done for all systems. This anomaly scan is done in every pregnant female, once AFI abnormalities were detected, search for a cause for this AFI abnormality.

In oligohydramnios \& anhydramnios: Maximum vertical pocket $<2 \mathrm{~cm}$, amniotic fluid index (AFI) $<$ $5 \mathrm{~cm}$.

In less than normal: AFI of 6-8 cm

The following questions should be answered in sequence: Is the UB full or empty? And is it distended or not? If kidneys are absent or not? And if present is it normal in size, echogenicity normal or not? Are they contain cysts or not?. Is there backpressure changes starting from the renal pelvis down the ureters till the UB? To exclude obstructive uropathy. Is IUGR, lung hypoplasia, Potter syndrome (pulmonary hypoplasia, Potter facies, club foot and contractures) present or not?

In polyhydramnios: (AFI) technique: Four pocket $\geq$ 25-cm.

Single largest pocket $\geq 8 \mathrm{~cm}$.

The seven questions of detailed fetal anatomy survey should be answered starting from the head down the fetal body caudally:

- Is there a CNS malformation is present?

- Is swallowed amniotic fluid obstructed before arriving in the esophagus?

- Is there heart failure?

- Is there chest causing abnormality?

- Is there a stomach bubble?

- Is there distal obstruction to cause fetal regurgitation?

- Is the overall tone is normal or not?

-3D \& 4D US: for all cases with associated congenital anomalies.

Regarding the placenta, umbilical cord: the diameter, the structure, presence of cysts or any anomaly 
(single artery), Umbilical artery RI to detect placental insufficiency.

Assessment of fetal maturity by both the traditional five parameters of assessing fetal maturity (lung echogenicity compared to the liver echogenicity, ossification centers, colonic haustra, liquor turbidity and the placental grading) and the newest ones(the lung volume, the lung perfusion and pulmonary artery RI).

Follow up the patients by the same technique of examination:

Every time the fetal biometry is reevaluated [to follow up the growth pattern and if small for date, IUGR, macrosomia were detected], fetal biophysical profile, AFV is reassessed for volume changes, turbidity, follow up of the maturity and the placental grading.

\section{Fetal outcome:}

The accuracy of diagnosis of congenital anomalies for completed pregnancies, was documented by post natal clinical examination done by a neonatologist to detect any congenital anomalies, to manage the survived babies, determine if they need any assisted ventilation, and determine if lung hypoplasia is present or not.

\section{Statistics:}

Using the statistical package for social sciences, version 20.0 (SPSS Inc., Chicago, Illinois, USA). Quantitative data were expressed as mean \pm standard deviation (SD). Statistical differences between groups were tested using Chi Square test for qualitative variables. P-values less than or equal to 0.05 were considered statistically significant. P-value $<0.001$ was considered highly significant. P-value $>0.05$ was considered insignificant.

\section{RESULTS}

(Table 1) shows that 80 females with amniotic fluid abnormalities were selected. The maternal age ranged from 19 to 40 years. The true gestational age ranged from 13 to 40 weeks. The corresponding US gestational age ranged from 12 to 39 weeks. They were classified into 3 groups.

\begin{tabular}{|l|c|c|}
\hline Groups & Number & Percentage \\
\hline $\begin{array}{l}\text { A. Oligohydramnios \& } \\
\text { anhydramnios }\end{array}$ & 34 & 42.5 \\
\hline B. Less than average & 8 & 10 \\
\hline C. Polyhydramnios & 38 & 47.5 \\
\hline \multicolumn{1}{|c|}{ Total number } & 80 & 100 \\
\hline
\end{tabular}

Table 1: Demonstrates the number \& percentage of each groups

Regarding different etiologies causing AF abnormalities, (Figures 1, 2 and 3) show that the congenital anomalies were common in the three groups.

We found the abnormalities associated with oligohydramnios as follows: Bilateral renal anomaly, renal agenesis and ureteropelvic junction obstruction, polycystic kidney disease, bladder outlet obstruction, Potter syndrome, narrow chest, CNS, heart, neck and MSK anomalies.

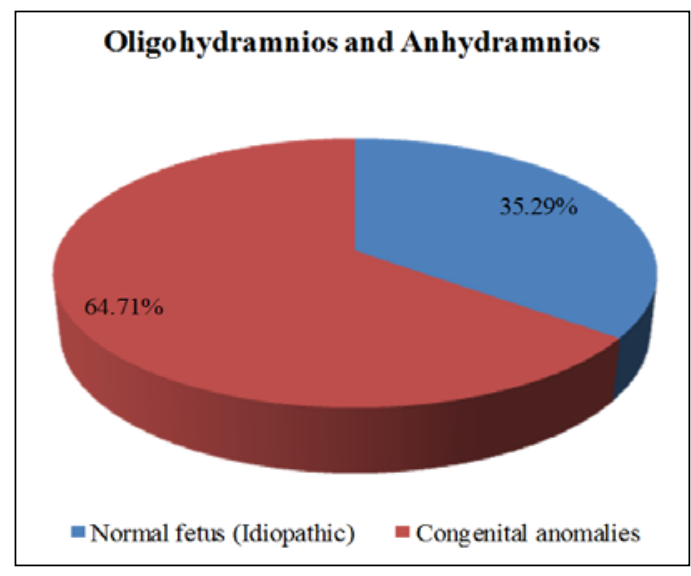

Fig 1: Pie chat demonstrates the incidence of different etiologies found in oligohydramnios and anhydramnios.

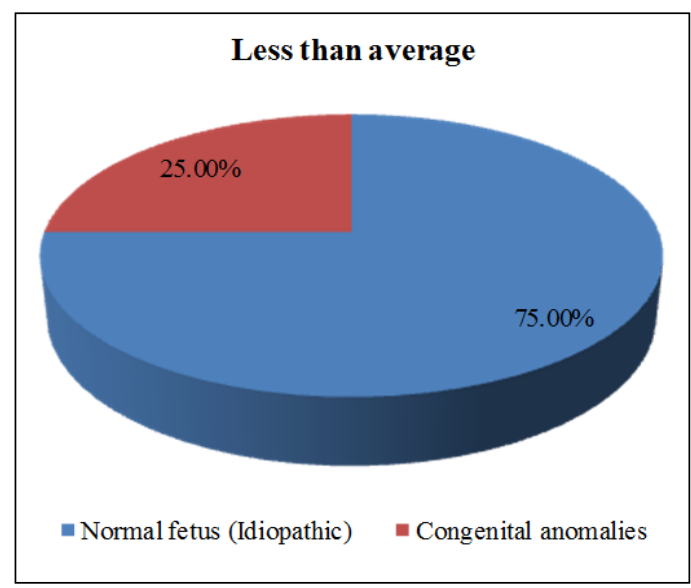

Fig 2: Demonstrates number \& percentage of different etiologies found in less than average AF.

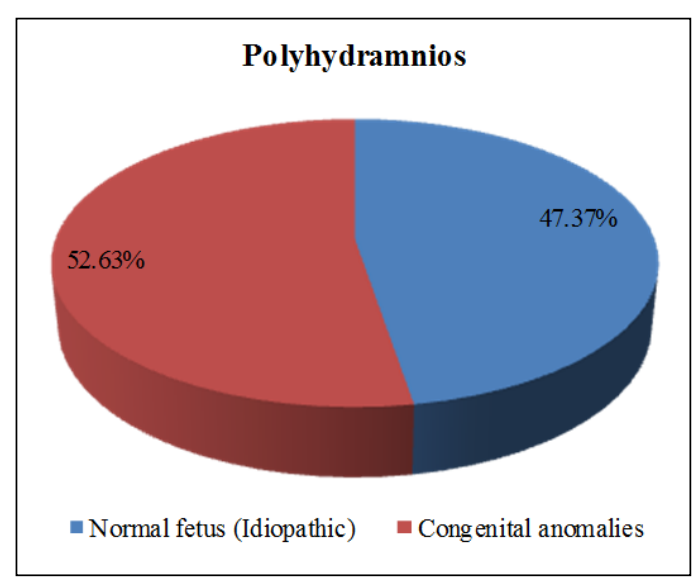


Fig 3: Demonstrates number \& percentage of different etiologies found in polyhydramnios.

We found the abnormalities associated with polyhydramnios as follows, Hydrops, Fetal Bowel Obstruction, Skeletal Dysplasia, CNS anomalies, Pulmonary Airway Malformation, Unilateral Ureteropelvic Junction Obstruction.

(Tables 2 and 3) show that the incidence of congenital anomalies was 44 cases out of 80 (55\%). Lethal anomalies was seen in 20 cases $(45.45 \%$ of congenital anomalies cases), non lethal in 24 cases (54.54\% of cong. anomalies cases).

The genitourinary abnormality is associated with oligohydramnios, of high significance, $\mathrm{P}$ - value (0.001). As well as Potter syndrome with a statistically significant P- value (0.001). Musculoskeletal (MSK) anomalies were associated with polyhydramnios with a statistically significant Pvalue $(0.03$.

Lung hypoplasia was detected in both oligo and polyhadramnios with a statistically insignificant Pvalue (0.073). The gastrointestinal (GIT) anomalies was associated with cases of polyhydramnios with a statistically nonsignificant P- value (0.175).

The CNS, heart are nearly equal with P- values $0.799,0.780$ respectively (statistically insignificant) (Figure 4)

According to the fetal biometric results $30 \%$ fetuses showed IUGR, 57.5\% fetuses showed normal development and 12, 5\% were macrosomic (Figures 4 and 5).

The IUGR: EFW $<$ and AC $<10$ th percentile + abnormal Dopplers.
LGA: Fetal weight $>90$ th percentile.

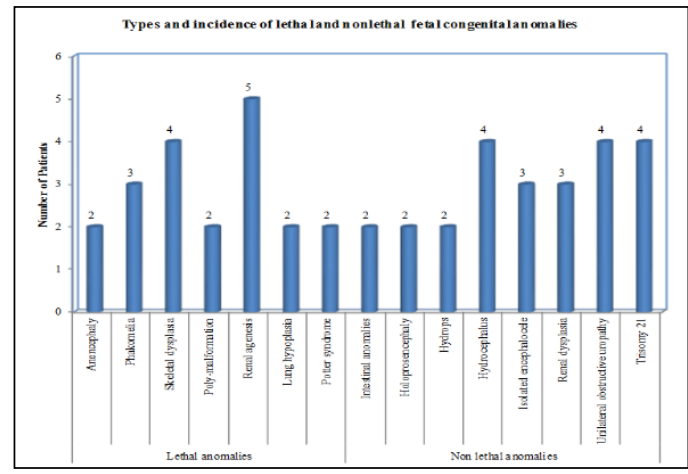

Fig 4: Types of lethal and nonlethal fetal congenital anomalies.

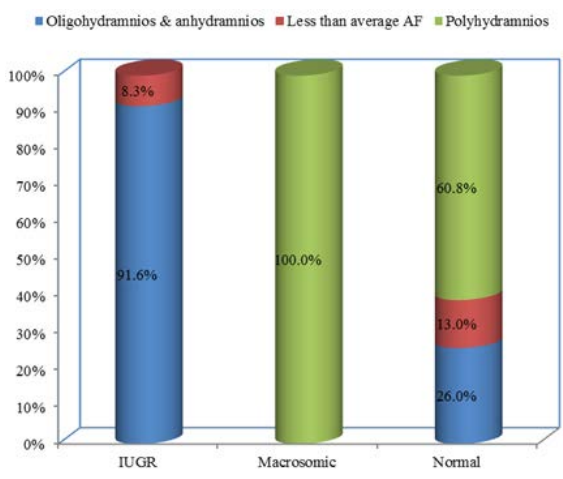

Fig 5: Illustrates the percentage of different growth potentials with different AFV.

\begin{tabular}{|l|l|l|l|}
\hline & Oligohyd.\& anhyd. & Polyhyd. & Less than average \\
\hline Normal fetus (Idiopathic) & $12(35.29 \%)$ & $18(47.37 \%)$ & $6(75 \%)$ \\
\hline Congenital anomalies & $22(64.71 \%)$ & $20(52.63 \%)$ & $2(25 \%)$ \\
\hline Total no. & $34(100.0 \%)$ & $38(100.0 \%)$ & $8(100.0 \%)$ \\
\hline Chi-square test & 29.635 & 24.495 & 0.571 \\
\hline p-value & $<0.001(\mathrm{HS})$ & $<0.001(\mathrm{HS})$ & 0.449 (NS) \\
\hline
\end{tabular}

Table 2: Demonstrates the percentage of congenital anomalies (p-value $>0.05 \mathrm{NS}$; p-value $<0.05 \mathrm{~S}$; p-value $<0.001 \mathrm{HS}$ ).

\begin{tabular}{|l|c|l|c|}
\hline Lethal anomalies & No. & \multicolumn{1}{|c|}{ Non lethal anomalies } & No. \\
\hline Anencephaly & 2 & Intestinal anomalies & 2 \\
\hline & & holoprosencephaly & 2 \\
\hline & & hydrops & 2 \\
\hline & & Hydrocephalus & 4 \\
\hline Phakomelia & 3 & Isolated encephalocele & 3 \\
\hline & & Renal dysplasia & 3 \\
\hline Skeletal dysplasia & 4 & & 4 \\
\hline Poly-malformation & 2 & Unilateral obstructive uropathy & \\
\hline Kidney agenesis & 5 & & 4 \\
\hline Lung hypoplasia & 2 & Trisomy 21 & \\
\hline Potter syndrome & 2 & & 24 \\
\hline Total & 20 & & \\
\hline
\end{tabular}

Table 3: Types and incidence of lethal and nonlethal fetal congenital anomalies. 


\section{DISCUSSION}

There are different causes of amniotic fluid changes, the causes of polyhydramnios are the idiopathic, macrosomia, DM, hydrops, twin-twin transfusion and bowel obstruction. ${ }^{6}$

The less common causes are arthrogryposis, skeletal dysplasia, placental chorioangioma \& pulmonary airway malformation. ${ }^{6}$

The causes of oligohydramnios are preterm pre-labor membrane rupture, urological abnormalities \& fetal growth retardation. ${ }^{6}$

Brace and Resnik ${ }^{1}$ found that the amniotic fluid abnormalities are about $7 \%$, polyhydramnios incidence is $1-2 \%$ of all pregnancies, Oligohydramnios is $4-5 \%$.

In our study $\mathrm{AF}$ problems occur in 80 pregnancies out of 3220 pregnant females and this represent about $2.48 \%$.

Biggo et al. ${ }^{2}$ had reported the incidence of polyhydramnios is $1 \%$. In our study, we found 38 cases of polyhydramnios which represent 1.18 percent.

We found 34 cases of oligohydramnios which represent 1.05 percent.

In 2007, Gabbe ${ }^{7}$ had studied the association between oligohydramnios and mortality rate.

In our study there were 9 fetuses with marked oligohydramnios or anhydramnios, 8 of them died (88.9 \%). 7 fetuses with marked mid pregnancy polyhydramnios, 4 of them died (57.1\%).

Rib \& colleagues ${ }^{3}$ searching in the different etiologies of oligohydramnios they found that, urological anomalies are the main causes apart from the maternal causes.

In our study we classified etiologies of oligohydramnios and anhydramnios group into, fetal congenital anomalies (64.71\%) which include renal and non renal anomalies, idiopathic (35.29\%)which most likely represent the placental insufficiency group and others.

In the less than average amniotic fluid group we found the following etiologies, idiopathic (75\%), congenital abnormalities and others (25\%).

In 2008, Standring et al. ${ }^{8}$ classified the etiology of polyhydramnios into idiopathic causes (35\%), DM (25\%), and fetal malformations (20\%).

Also, Biggio at al. ${ }^{2}$ found that the etiology of polyhydramnios include macrosomia, hydrops, ascites, pleural effusions, and GIT obstruction tract, MSK abnormalities.
In this study we classified etiologies of polyhydramnios into, fetal congenital anomalies (52.64\%), idiopathic (47.36\%). we had found the malformations associated with the polyhydramnios as follows: GIT, MSK, neck, face, heart, hydrops fetalis and CNS anomalies.

In 2007 Gabbe, ${ }^{7}$ revealed that oligohydramnios, can cause IUGR, morbidity and mortality.

In this study there were 22 fetuses with oligohydramnios or anhydramnios complicated by IUGR, 8 of them died (36.36\%).

In 2008, Laudy and Wladimiroff ${ }^{9}$ reported that pulmonary hypoplasia can cause respiratory failure and mortality.

In our study, we found positive association between lung hypoplasia, oligo, polyhydramnios, congenital anomalies and different growth abnormalities.

The differentiation between lethal and nonlethal pulmonary hypoplasia were nonspecific because of the small sample who completed the pregnancy.

The five traditional parameters; lung echogenicity / the liver echogenicity, colonic hustra, ossification centers, liquor turbidity and placental grading, predicting pulmonary hypoplasia are of high sensitivity but of very low specificity in predicting pulmonary abnormal size. The new three parameters of predicting lung hypoplasia, which are the pulmonary artery RI, lung perfusion and lung volume were found to be good predictors. ${ }^{10}$

In our study, there were statistically significant relation between the amniotic fluid abnormalities, congenital anomalies and unfavorable outcomes.

\section{CONCLUSION}

Despite significant percentage of idiopathic etiology of fluid abnormality, we concluded that congenital anomalies should be carefully searched on whenever fluid abnormalities were detected. Full anomaly scan and close antenatal follow up are recommended.

The $4 \mathrm{D}$ ultrasound is a method alternative to $2 \mathrm{D} \&$ $3 \mathrm{D}$ ultrasonography in the survey of congenital malformations. 2D also is important in assessment of lung maturity for detection of method of delivery or CS.

In the study, 2D volume measurements, is a good tool for antenatal prediction of fetal wellbeing \& follow up.

The result of the study shows a strong positive correlation between fetal wellbeing and amniotic fluid abnormalities.

Larger studies are necessary to confirm these results.

\section{(1)}


The early diagnosis of pregnancies at risk for preventable perinatal handicap is a primary goal of the obstetric care providers. One of the most consistently used justifications for requesting obstetric ultrasound is early diagnosis of any obstetric abnormality as amniotic fluid abnormalities and any associated fetal anomalies before delivery which can provide a number of management options.

In our study we concluded that, when oligohydramnios is associated with IUGR, fetal congenital anomalies, or pulmonary hypoplasia, this will contribute to high morbidity and mortality rate.

Also, we found that severe polyhydramnios is associated with congenital malformations.

\section{REFERENCES}

1. Brace RA and Resnik R. Dynamics and Disorders of Amniotic Fluid. Maternal-Fetal Medicine. 4th ed. 1999; 632-43.

2. Biggio JR Jr, Wenstrom KD, Dubard MB, et al. Hydramnios prediction of adverse perinatal outcome".Obstet,Gynecol Nov; 1999; 94(5): 7737.

3. Rib DM, Sherer DM, and Woods JR Jr. Maternal and neonatal outcome associated with prolonged premature rupture of membranes below 26 weeks' gestation. Am J Perinatol. 1993; 10(5): 369-73.

4. Roland LB and Brian SC. Polyhydramnios and Oligohydramnios. emedicine up dated, 2006.

5. Phelan JP, Ahn MO, and Smith CV. Amniotic fluid index measurement during pregnancy. $J$ Report Med. 1987; 32(8): 601-4.

6. Woodward PJ, Kennedy A, Sohaey R, et al Diagnostic imaging: Obestetric. Amirsys, Elsevier, third edition, textbook. 2017; (15):1028: 52.

7. Gabbe SG, Jennifer R. Niebyl, et al. Obstetrics normal and problem pregnancies. Fifth Edition, Churchill Livingstone Elsevier, Philadelphia, 2007.

8. Standring S., Harold E., Jeremiah C, et al. Gray's anatomy, Female reproductive system, fetal membranes. 2008; 105:1340.

9. Laudy JA and Wladimiroff JW. The fetal lung. 2: Pulmonary hypoplasia. Ultrasound Obstet Gynecol. 2000; 16:482-94.

10. McGahan P.J. and Goldberg B.Bl. Diagnostic ultrasound. Lippincott- Raven, 1st edition, Biblio Med textbook, 1997. 